\title{
Quitones (Mollusca: Polyplacophora) de EI Salvador, América Central
}

\author{
Cedar I. García-Ríos ${ }^{1}$, Migdalia Álvarez-Ruiz ${ }^{2}$, José E. Barraza ${ }^{3}$, Ana M. Rivera ${ }^{4}$ \& \\ Carlos R. Hasbún ${ }^{5}$ \\ 1 Departamento de Biología, Universidad de Puerto Rico en Humacao, Humacao, Puerto Rico 00792; cgarcia@www. \\ uprh.edu \\ 2 Departamento de Biología, Universidad de Puerto Rico en Ponce, Ponce, Puerto Rico 00732; biomar@coqui.net \\ 3 Ministerio de Medio Ambiente y Recursos Naturales, San Salvador, El Salvador; jenriquebarr@integra.com.sv \\ 4 Museo de Historia Natural, San Salvador, El Salvador; anadararivera@yahoo.com

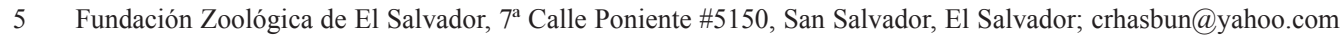

\author{
Recibido 13-IX-2002. Corregido 27-IX-2005. ～Aceptado 28-X-2006.
}

\begin{abstract}
Chitons (Mollusca: Polyplacophora) from El Salvador, Central America. Collections of 11 species of shallow water Polyplacophora from El Salvador were made in July 2002. Previously only five species had been documented in El Salvador: Chaetopleura lurida (Sowerby, 1832); Ischnochiton guatemalensis (Thiele, 1910); Ceratozona angusta (Thiele, 1909); Chiton stokesii (Broderip, 1832) and Acantochitona exquisita (Pilsbry, 1893). Of these, I. guatemalensis and A. exquisita were not collected in this census. Seven other species are reported here for El Salvador for the first time: Lepidochitona beanii (Carpenter, 1857); Ischnochiton dispar (Sowerby, 1832); Stenoplax limaciformis (Sowerby, 1832); Callistochiton expressus (Carpenter, 1865); Acanthochitona arragonites (Carpenter, 1867); A. ferreirai (Lyons, 1988) and A. hirudiniformis (Sowerby, 1832). The known geographic distribution of $I$. dispar is extended to the north. An un-named species of Lepidochitona is briefly described. Rev. Biol. Trop. 55 (1): 171-176. Epub 2007 March. 31.
\end{abstract}

Key words: Polyplacophora, chitons, El Salvador, records, Central America.

El conocimiento de la fauna de quitones (Mollusca: Polyplacophora) de las aguas litorales de El Salvador es limitado. Anteriormente a este trabajo solo cinco especies estaban documentadas para estas aguas. Estas son: Ischnochiton guatemalensis (Thiele, 1910) localidad del tipo (Slieker 2000); Chaetopleura lurida (Sowerby, 1832) en Kaas y Van Belle (1987); Ceratozona angusta (Thiele, 1909) en Keen (1971); Chiton stokesii (Broderip, 1832) en Bullock (1988) y Hernández (1992) y Acantochitona exquisita (Pilsbry, 1893) en Watters (1990).

Aquí se documenta la presencia de otras especies de quitones en El Salvador, ampliamos un ámbito de distribución geográfica $\mathrm{y}$ describimos una especie innominada de Lepidochitona.

\section{MATERIALES Y MÉTODOS}

Se realizaron nueve viajes de colección a siete zonas rocosas diferentes en julio del 2002 (Cuadro 1 y Fig. 1). La latitud y longitud de las estaciones se determinó usando el sistema de posicionamiento global (GPS) marca Magellan modelo 315. En todas las estaciones se recolectó a mano, examinando la superficie expuesta de las rocas, así como la superficie enterrada o en sombras. Solo en las islas Pirigallo y Martín Pérez en el Golfo de Fonseca se usó equipo de buceo autónomo y se examinaron rocas hasta los 4 metros de profundidad. En las demás estaciones se recolectó durante los períodos de marea baja y en profundidades menores a un metro (bajo el nivel de marea baja). Se realizó un mayor esfuerzo en las localidades y 


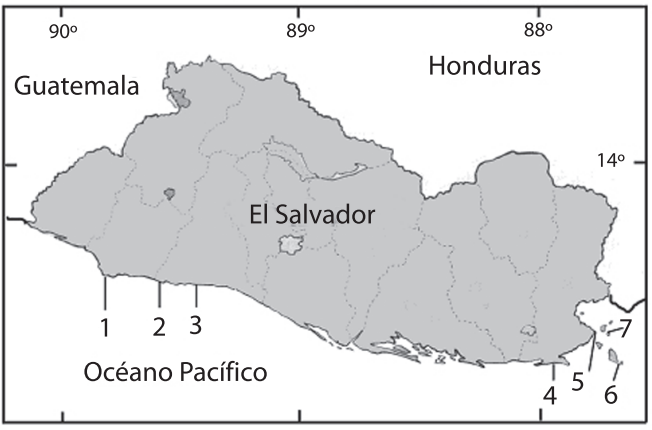

Fig. 1. Estaciones de recolección de quitones en El Salvador: 1- Los Cóbanos, 2- El Pital, 3- El Zonte, 4- Playa Maculís, 5- Punta Chiquirín, 6- Isla Pirigallo y 7- Isla Martín Pérez.

Fig. 1. Collection stations in El Salvador: 1- Los Cóbanos, 2- El Pital, 3- El Zonte, 4- Playa Maculís, 5- Punta Chiquirín, 6- Isla Pirigallo y 7- Isla Martín Pérez. situaciones ambientales que presentaron mayor diversidad.

Los lotes de quitones coleccionados fueron relajados y aplanados para su preservación usando alcohol etílico diluido con agua de mar al $70 \%$. Los ejemplares seleccionados para ser examinados con el microscopio electrónico de barrido (MEB) se prepararon utilizando el método descrito en Bullock (1988). La identificación de los quitones se realizó utilizando la serie de monografías taxonómicas de Kaas y Van Belle (1985, 1987, 1990 y 1994) y Keen (1971). La clasificación taxonómica se presenta utilizando el esquema de clasificación de Kaas y Van Belle (1998).

Los ejemplares que resultaron de estas colecciones fueron depositados en varias

CUADRO 1

Localización de las estaciones y descripción de hábitat de los quitones recoleccióndos en El Salvador

TABLE 1

Location of stations nand habitat descriptions for chiton collection sites in El Salvador

\begin{tabular}{|c|c|c|c|c|}
\hline Lugar & Departamento & Latitud & Longitud & Tipo de hábitat \\
\hline Los Cóbanos & Sonsonate & $13^{\circ} 31^{\prime} 40^{\prime \prime}$ & $89^{\circ} 48^{\prime} 44$ & $\begin{array}{l}\text { Rocas sueltas sobre fondo de arenas gruesas entre } \\
0 \text { y } 1 \text { metro de profundidad bajo el nivel de marea } \\
\text { baja. Abundante crecimiento de algas rojas e } \\
\text { invertebrados sésiles en el sublitoral. }\end{array}$ \\
\hline El Pital & La Libertad & $13^{\circ} 30^{\prime} 41^{\prime \prime}$ & 89³6’13”' & $\begin{array}{l}\text { Costa rocosa (aglomerados cementados), en pozas } \\
\text { de marea formadas en el mesolitoral y bajo rocas } \\
\text { sueltas en el sublitoral alto ( } 0.5 \text { profundidad bajo } \\
\text { nivel de marea baja). Crecimiento de mitílidos, } \\
\text { cirrípedos y algas, incluyendo filamentosas. }\end{array}$ \\
\hline El Zonte & La Libertad & $13^{\circ} 29^{\prime} 42^{\prime \prime}$ & $89^{\circ} 26^{\prime} 11^{\prime \prime}$ & $\begin{array}{l}\text { Costa similar a El Pital. También se colectó en } \\
\text { costa de guijarros (canto rodado) sueltos sobre } \\
\text { arenas negras a menos de } 1 \text { metro de profundidad } \\
\text { bajo el nivel de marea baja. }\end{array}$ \\
\hline Playa Maculís & La Unión & $13^{\circ} 09^{\prime} 30^{\prime \prime}$ & $89^{\circ} 55^{\prime} 24^{\prime \prime}$ & $\begin{array}{l}\text { Agregado de rocas sueltas perpendiculares a playa } \\
\text { de arenas negras. Crecimiento de algas pardas } \\
\text { (Padina) y esponjas. }\end{array}$ \\
\hline Punta Chiquirín & La Unión & $13^{\circ} 17^{\prime} 21^{\prime \prime}$ & $89^{\circ} 47^{\prime} 18^{\prime \prime}$ & $\begin{array}{l}\text { Costa de guijarros. Crecimiento de cirrípedos y una } \\
\text { abundante cantidad de cangrejos pequeños. }\end{array}$ \\
\hline Isla Pirigallo & $\begin{array}{l}\text { La Unión: } \\
\text { Golfo de Fonseca }\end{array}$ & $13^{\circ} 10^{\prime} 26^{\prime \prime}$ & $87^{\circ} 41^{\prime} 26^{\prime \prime}$ & $\begin{array}{l}\text { Grandes rocas acumuladas en las orillas y rocas } \\
\text { sueltas, visibilidad menor a un metro. Crecimiento } \\
\text { abundante de hidrozoarios. }\end{array}$ \\
\hline Isla Martín Pérez & $\begin{array}{l}\text { La Unión: } \\
\text { Golfo de Fonseca }\end{array}$ & $13^{\circ} 16^{\prime} 57^{\prime \prime}$ & $87^{\circ} 44^{\prime} 45^{\prime \prime}$ & Similar a la Isla Pirigallo. \\
\hline
\end{tabular}


instituciones: el Museo de Historia Natural de El Salvador (MHNES), la Universidad de El Salvador y en la Colección Biológica de la Universidad de Puerto Rico en Humacao (CBUPRH).

\section{RESULTADOS}

Se recolectaron 421 ejemplares que se clasificaron en 11 especies diferentes (Cuadro 2). De la totalidad de especímenes recolectados, C. stokesii e Ischnochiton dispar fueron las especies más comunes. C. stokesii se documentó en todas las estaciones muestreadas y es la especie mas común en todo el mesolitoral. La especie I. dispar se recolectó en seis de las siete estaciones y resultó muy abundante en el mesolitoral bajo y el sublitoral alto. Otras especies localmente abundantes son: C. lurida, Callistochiton expressus, Acanthochitona ferreirai y Stenoplax limaciformis. Estas últimas cuatro especies siempre fueron encontradas bajo rocas parcialmente enterradas en la arena, en la parte alta del sublitoral.

Las comunidades de quitones de Los Cóbanos y la Playa Maculís fueron las de mayor diversidad. En ambos lugares la cobertura y diversidad aparente de la fauna y flora asociadas al sustrato rocoso fue mayor.

De las once especies identificadas en este trabajo, siete son informes nuevos para El Salvador: Lepidochitona beanii (Carpenter 1857); I. dispar (Sowerby 1832) (Fig. 2); S. limaciformis (Sowerby 1832); C. expressus (Carpenter 1865); Acanthochitona arragonites (Carpenter 1867); A. ferreirai (Lyons 1988) (Fig. 3) y Acanthochitona hirudiniformis (Sowerby 1832).

Dos ejemplares de una especie sin describir del género Lepidochitona (Fig. 4) fueron recolectadas. Los ejemplares presentan diferencias morfológicas con L. beanii, la única especie de ese género documentada para la zona, que también fue recolectada. Lepidochitona sp. es diferente a otras especies del género en las proporciones de las dimensiones de las placas,

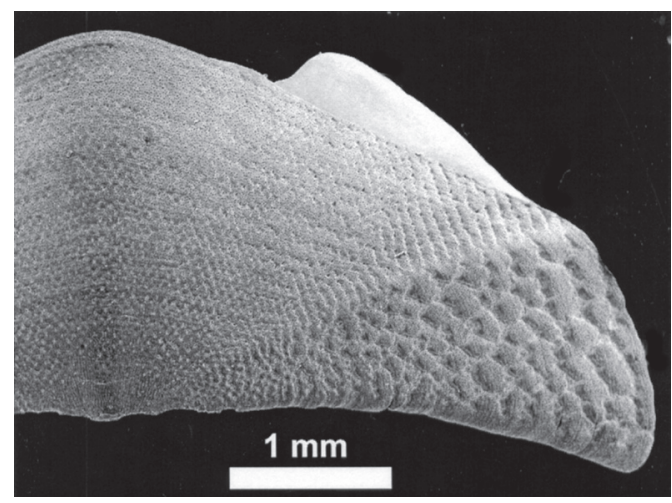

Fig. 2. Placa intermedia de Ischnochiton dispar (ejemplar CBUPRH \# 1943). Los Cóbanos, Sonsonate, El Salvador Costa rocosa, bajo rocas sueltas parcialmente enterradas en arena, sublitoral alto (-0.3 a $-1.0 \mathrm{~m}$ durante marea baja). Col. M. Álvarez-Ruiz, C. I. García-Ríos y A.M. Rivera 26 de julio de 2002 .

Fig. 2. Intermediate valve of Ischnochiton dispar (SEM photo, CBUPRH \# 1943). Los Cóbanos, Sonsonate, El Salvador Rocky shore, under stones partially buried in sand, 0.3 to $-1.0 \mathrm{~m}$ depth at low tide. Col. M. Álvarez-Ruiz, C. I. García-Ríos y A.M. Rivera July 26, 2002.

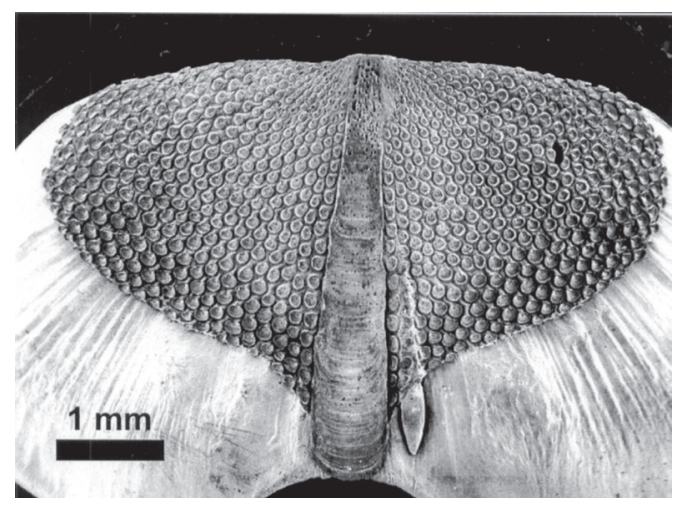

Fig. 3. Placa intermedia de Acanthochitona ferreirai, con pústulos apiñados radiando del ápice de la placa y la extensión del tegumento (ejemplar CBUPRH \# 1942). Los Cóbanos, Sonsonate, El Salvador Costa rocosa, bajo rocas sueltas parcialmente enterradas en arena, sublitoral alto (0.3 a -1.0 m durante marea baja). Col. M. Álvarez-Ruiz, C. I. García-Ríos y A.M. Rivera 26 de julio de 2002.

Fig. 3. Intermediate valve of Acanthochitona ferreirai, with crowded pustules radiating from the apex and the extension of the tegmentum (SEM photo, CBUPRH \# 1942). Los Cóbanos, Sonsonate, El Salvador Rocky shore, under stones partially buried in sand, 0.3 to $-1.0 \mathrm{~m}$ depth at low tide. Col. M. Álvarez-Ruiz, C. I. García-Ríos y A.M. Rivera July 26, 2002. 


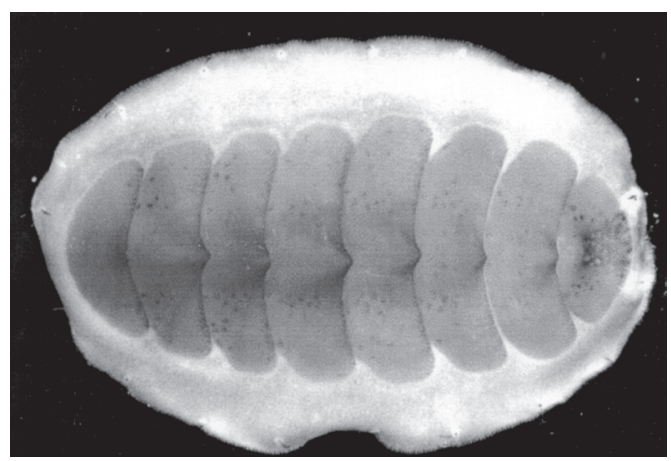

Fig. 4. Lepidochitona sp. (CBUPRH \# 1930). El Pital, La Libertad, El Salvador. 7.8 X $5.2 \mathrm{~mm}$.Costa rocosa, zona superior del sublitoral, en roca suelta que tenía superficie cubierta por alga roja, durante marea baja. Col. M. ÁlvarezRuiz, J.E. Barraza, C.I. García-Ríos y A.M. Rivera. 24 de julio de 2002. Descripción: Pequeños, cerca de $8 \mathrm{~mm}$ de longitud. Las placas intermedias centrales son más anchas que las intermedias cercanas a las placas terminales, lo que acentúa una apariencia ovalada. Tegumento y cinturón de color rojo oscuro cuando están vivos, el tegumento se torna más anaranjado en alcohol y el cinturón se torna marrón. Puede tener marcas claras en algunas de las placas. El tegumento tiene superficie suave. El cinturón está cubierto por espículas diminutas que le dan aspecto granular. Cerca del borde exterior del cinturón y distribuidas en forma regular tiene otras espículas largas, transparentes y arqueadas.

Fig. 4. Lepidochitona sp. (CBUPRH \# 1930). El Pital, La Libertad, El Salvador. 7.8 X $5.2 \mathrm{~mm}$. Col. M. ÁlvarezRuiz, C. I. García-Ríos y A.M. Rivera. July 24, 2002. Description: Small, near $8 \mathrm{~mm}$ in length. The III, IV and V intermediate valves are wider than those near the terminal valves, which accentuates the oval appearance. Tegmentum and girdle of the live chiton dark red, in alcohol the tegmentum becomes orange and the girdle brownish. Whitish marks in some valves. The tegmentum is smooth. The girdle is wide, dorsally covered with minute bluntly pointed spicules that give a granular aspect. Small tufts of long, curved hyaline needles with a regular distribution near the outer edge of the girdle.

los elementos del cinturón y la escultura del tegumento.

\section{DISCUSIÓN}

Las trece especies informadas para El Salvador pertenecen a ocho géneros y representan tres familias. Corresponde al $65 \%$ de la fauna de quitones esperada, si consideramos las otras especies del litoral, informadas
CUADRO 2

Sinopsis taxonómica de los quitones

(Mollusca: Polyplacophora) de El Salvador

TABLE 2

Taxonomic synopsis of chitons

(Mollusca: Polyplacophora) from El Salvador

Clase: POLYPLACOPHORA Gray, 1821

Orden: NEOLORICATA Bergenhayn, 1955

Suborden: ISCHNOCHITONINA Bergenhayn, 1930

Familia: ISCHNOCHITONIDAE Dall, 1889

Subfamilia: Lepidochitonninae Iredale, 1914

Género: Lepidochitona Gray, 1821

Lepidochitona beanii (Carpenter, 1857)

Lepidochitona sp.

Subfamilia: Chaetopleurinae

Género: Chaetopleura Shuttleworth, 1853

Chaetopleura lurida (Sowerby, 1832)

Subfamilia: Ischnochitoninae

Género: Ischnochiton Gray, 1847

Ischnochiton dispar (Sowerby, 1832)

Ischnochiton guatemalensis Thiele, 1910

(no encontrada en este censo)

Género: Stenoplax Dall, 1879

Stenoplax limaciformis (Sowerby, 1832)

Subfamilia: Callistoplacinae Pilsbry, 1893

Género: Callistochiton Dall, 1879

Callistochiton expressus (Carpenter, 1865)

Género: Ceratozona Dall, 1882

Ceratozona angusta Thiele, 1909

Familia: CHITONIDAE Rafinesque, 1815

Subfamilia: Chitoninae

Género: Chiton Linnaeus, 1758

Chiton stokesii Broderip, 1832

Suborden: ACANTHOCHITONINA Bergenhayn, 1930

Familia: ACANTHOCHITONIDAE Pilsbry, 1893

Subfamilia: Acanthochitoninae

Género: Acanthochitona Gray, 1821

Acanthochitona arragonites (Carpenter, 1867)

Acantochitona exquisita (Pilsbry, 1893)

(no encontrada en este censo)

Acanthochitona ferreirai Lyons, 1988

Acanthochitona hirudiniformis (Sowerby, 1832)

en países al norte y al sur de El Salvador. Esas especies son: Chaetopleura unilineata (Leloup, 1954); Stenoplax rugulata (Sowerby, 1832); Lepidozona allynsmithi (Ferreira, 
1974); Callistochiton elenensis (Sowerby, 1832); Callistoplax retusa (Sowerby, 1832); Acanthochitona angelica (Dall, 1919) y A. imperatrix (Watters, 1981).

I. dispar (Fig. 2) está informada para varias localidades en Panamá, Costa Rica y Nicaragua (Kaas y Van Belle 1990). El presente informe extiende su distribución conocida hacia el norte. En forma similar Lyons (1988) en la descripción de $A$. ferreirai, limita su distribución a la costa Pacífica de Costa Rica y Panamá. La foto del MEB (Fig. 3) permite identificar a nuestros ejemplares como $A$. ferreirai (extendiendo su distribución al norte) y distinguirlos de $A$. rhodea. En una revisión de los miembros de la familia Acanthochitonidae del Caribe Lyons (1988) describe a A. ferreirai como una especie de la región Panámica, muy relacionada (y similar) con $A$. hemphilli y $A$. rhodea de la región del Caribe. De ser exacta esta revisión probablemente se corrobore que todos los informes de $A$. rhodea en el Pacífico corresponden a la especie $A$. ferreirai.

Lepidochitona sp. de El Salvador es la segunda especie del género reconocida para la provincia Panámica. La única especie previamente conocida es L. beanii (Carpenter, 1857). Lepidochitona $\mathrm{sp}$ se diferencia de L. beanii en poseer espículas hialinas más largas, un tegumento suave, placas intermedias con márgenes laterales redondeados y espículas marginales más pequeñas (Fig. 4).

Estudios de bioquímica comparada realizados en miembros de la familia Lepidochitonidae por Eernisse (2004) proponen reconocer tres clados dentro de la familia Lepidochitonidae: uno que agrupe a los Lepidochitona spp. del norte de Europa (se mantendrían en el género Lepidochitona); otro que agrupe a los del Pacífico norte (en el género Cyanoplax.) y el tercero que agruparía a las especies caribeñas, la Panámica y las del sur de África con Nuttallina spp (el autor no sugiere clasificación de género en este clado). En la designación del género nos basamos estrictamente en los caracteres morfológicos, según el esquema de Kaas y Van Belle (1998). Lepidochitona sp. fue clasificada en ese género por la presencia de espículas hialinas en el cinturón. Se descartó el género Mopalia, pues nuestros ejemplares no tienen cerdas y Dendrochiton (o Lepidochitona) porque sus espículas no están ramificadas.

Los ejemplares de Lepidochitona sp. encontrados en El Salvador son morfológicamente muy similares a L. liozonis del Caribe. Además, a ambas especies las encontramos en asociación con algas coralinas crustosas (Corallinaceae: Neogoniolithon spp.). En ambas el quitón vivo presenta una coloración muy similar al alga con que se asocia, como se ilustra en Littler y Littler (2000). La capacidad de atenuar o intensificar la coloración fue observada en el campo, al examinar los ejemplares vivos. La especie de Lepidochitona encontrada en El Salvador puede considerarse especie hermana de L. liozonis.

\section{AGRADECIMIENTOS}

Agradecemos a las instituciones a las que estamos adscritos por el tiempo y el apoyo logístico para este trabajo. El transporte fue pagado por la Alianza para el Adelanto de la Enseñanza Universitaria en Puerto Rico. El Fondo para la Investigación de la Universidad de Puerto Rico en Humacao (UPR-H) apoyó al primer autor. Agradecemos a Ernesto López (Ministerio de Ambiente y Recursos Naturales de El Salvador) el permiso de recolección y el uso del microscopio electrónico de barrido al Departamento de Física, UPR-H, Programa Collaborative to Integrate Research and Education (Nacional Science Foundation Grant \#9872689).

\section{RESUMEN}

En El Salvador se habían registrado los poliplacóforos Chaetopleura lurida (Sowerby, 1832); Ischnochiton guatemalensis (Thiele, 1910); Ceratozona angusta (Thiele, 1909); Chiton stokesii (Broderip, 1832) y Acantochitona exquisita (Pilsbry, 1893). Recolectamos quitones en aguas someras de El Salvador en julio del 2002, agragando a la lista a Lepidochitona beanii (Carpenter, 1857); Ischnochiton dispar (Sowerby, 1832); Stenoplax limaciformis 
(Sowerby, 1832); Callistochiton expressus (Carpenter, 1865); Acanthochitona arragonites (Carpenter, 1867); Acanthochitona ferreirai (Lyons, 1988) y Acanthochitona hirudiniformis (Sowerby, 1832). Ampliamos la distribución documentada de $I$. dispar hacia el norte y describimos brevemente una especie innominada de Lepidochitona.

Palabras clave: Polyplacophora, quitones, El Salvador, registros, América Central.

\section{REFERENCIAS}

Bullock, R.C. 1988. The genus Chiton in the new world (Polyplacophora: Chitonidae). Veliger 31: 141-191.

Eernisse, D.J. 2004. Revival of the genus Cyanoplax Pilsbry, 1892 for a clade of West Coast chitons [abstract], p. 33-35. In J.C. Martínez and R.V. Yeomans (eds.) Program and Abstracts of the 37th Annual Meeting of the Western Society of Malacologists, Ensenada, Baja California, México.

Hernández, M.A. 1992. Inventario preliminar de moluscos marinos de la costa de El Salvador. Secretaría Ejecutiva del Medio Ambiente, San Salvador, El Salvador. $172 \mathrm{p}$.

Littler D.S. \& M.M. Littler. 2000. Caribbean Reef Plants: an identification guide to the reef plants of the Caribbean, Bahamas, Florida and Gulf of Mexico. Offshore Graphic, Washington DC. 542 p.

Lyons, W.G. 1988. A review of caribbean Acanthochitonidae (Mollusca: Polyplacophora) with descriptions of six new species of Acanthochitona Gray, 1821. Am. Malac. Bull. 6: 79-114.
Kaas, P. \& R.A. Van Belle. 1985. Monograph of living chitons. Vol. 2, Suborder Ischnochitonina, Ischnochitonidae: Schizoplacinae, Callochitoninae \& Lepidochitoninae. E.J. Brill / W. Backhuys, Leiden, Holanda. 198 p.

Kaas, P. \& R.A. Van Belle. 1987. Monograph of living chitons. Vol. 3, Ischnochitonidae: Chaetopleurinae, Ischnochitoninae (pars). E.J. Brill / W. Backhuys, Leiden, Holanda. 302 p.

Kaas, P. \& R.A. Van Belle. 1990. Monograph of living chitons (Mollusca: Polyplacophora), Vol. 4, Suborder Ischnochitonina: Ischnochitonidae: Ischnochitoninae (continued), additions to vols. 1, 2 y 3. E.J. Brill, Leiden, Holanda. 298 p.

Kaas, P. \& R.A. Van Belle. 1994. Monograph of living chitons (Mollusca: Polyplacophora), Vol. 5, Suborder Ischnochitonina: Ischnochitonidae (concluded); Callistoplacinae, Mopalidae, additions to vols. 1-4. E.J. Brill, Leiden, Holanda. 402 p.

Kaas, P. \& R.A. Van Belle. 1998. Catalogue of living chitons (Mollusca: Polyplacophora, Backhuys Publishers, Leiden, Holanda. 204 p.

Keen, A.M. 1971. Sea shells of tropical West America: marine mollusks from Baja California to Peru. Stanford University, Stanford, California, EEUU. 1064 p.

Slieker, F.J.A. 2000. Chitons of the world: an illustrated synopsis of recent Polyplacophora. L'Informatore, Piceno, Italia. 154 p.

Watters, G.T. 1990 A review of the recent eastern Pacific Acanthochitoninae (Mollusca: Polyplacophora: Cryptoplacidae) with the description of a new genus, Americhiton. Veliger 33: 241-271. 